\title{
Optimistic belief updating despite inclusion of positive events
}

DOI:

10.1016/j.Imot.2017.05.001

Document Version

Accepted author manuscript

Link to publication record in Manchester Research Explorer

\section{Citation for published version (APA):}

Marks, J., \& Baines, S. (2017). Optimistic belief updating despite inclusion of positive events. Learning and Motivation, 58, 88-101. https://doi.org/10.1016/j.Imot.2017.05.001

\section{Published in:}

Learning and Motivation

\section{Citing this paper}

Please note that where the full-text provided on Manchester Research Explorer is the Author Accepted Manuscript or Proof version this may differ from the final Published version. If citing, it is advised that you check and use the publisher's definitive version.

\section{General rights}

Copyright and moral rights for the publications made accessible in the Research Explorer are retained by the authors and/or other copyright owners and it is a condition of accessing publications that users recognise and abide by the legal requirements associated with these rights.

\section{Takedown policy}

If you believe that this document breaches copyright please refer to the University of Manchester's Takedown Procedures [http://man.ac.uk/04Y6Bo] or contact uml.scholarlycommunications@manchester.ac.uk providing relevant details, so we can investigate your claim.

\section{OPEN ACCESS}


Optimistic belief updating despite inclusion of positive life events

Joseph Marks ${ }^{1,2}$ \& Stephanie Baines ${ }^{1,3}$

${ }^{1}$ Department of Experimental Psychology, University College London, London, UK

${ }^{2}$ Influence At Work, 4 Kinsbourne Court, 96-100 Luton Road, Harpenden, UK

${ }^{3}$ Division of Cognitive Neuroscience and Experimental Psychology, Faculty of Biology, Medicine and Health, University of Manchester, UK

* Corresponding Author (joseph@influenceatwork.co.uk) 


\begin{abstract}
A recent methodology - namely, the belief update task - used in the study of unrealistic optimism has provided a mechanistic account of how people maintain positive illusions about their future in the face of disconfirming evidence. This methodology has been used in a series of neuroscientific studies and neural moderators of unrealistic optimism have been established. A subsequent critique of the belief update task by Shah et al. (2016) has cast doubt over the validity of these findings however, with the authors instead suggesting that apparent optimistic belief updating is in fact a statistical artefact resulting from a flawed methodology. Specifically, Shah et al. assert that the inclusion of positive events in the belief update task can help test the validity of the optimistic account of belief updating, while proponents have suggested that caution should be taken when adapting this task to study positive life events because there is a lack of accurate information regarding the likely frequency of such events. Using a subset of the life events used by Shah et al., the current paper demonstrates that optimistic belief updating should still be observed when positive life events are included in the belief update task.
\end{abstract}

Keywords: Optimism Bias; Belief Updating; Positive Events; Unrealistic Optimism; Motivated Reasoning; Future Projection 


\section{Introduction}

In everyday life, people are often faced with decisions that require them to estimate the likelihood of certain events happening to them in the future (Tversky \& Kahneman, 1974). Folk-wisdom dictates that knowledge is power and, indeed, the classical view of human judgment predicts that future projections should rely on accurate, objective and unbiased evidence (e.g. Trope, 1980). However, this normative account has been challenged by a second school of academic thought, one that takes a cognitive-affective perspective of judgement formation. This school has suggested that an optimism bias is a vital component of healthy psychological functioning (Garrett et al., 2014; Korn, Sharot, Walter, Heekeren, \& Dolan, 2014; Strunk, Lopez, \& DeRubeis, 2006; Taylor \& Brown, 1988; Tyler \& Rosier, 2009) and that humans have a pervasive tendency to make systematically biased probability assessments when estimating personal risks (Sharot, 2011; Sharot, 2012). This line of reasoning suggests that people ultimately ignore warning signs in order to maintain positive hedonic feelings and, consequently, that human cognition acts in a manner more aligned to the philosophy ignorance is bliss.

A few recent papers have critiqued the evidential basis for unrealistic optimism and cast some doubt over the validity of the methodologies used to study both comparative and absolute optimism (Harris \& Hahn, 2011; Harris, Shah, Catmur, Bird, \& Hahn, 2013; Shah, Harris, Hahn, Catmur, \& Bird, 2016). If accurate, this account of the literature is worrisome given the use of unrealistic optimism in explaining a number of behavioural effects that occur in applied settings, including health (Dillard, McCaul, \& Klein, 2006; Jansen et al., 2011; Shepherd, 2002; van der Velde, Hooykas, \& van der Joop, 1992; van der Velde, van der Joop, \& Hooykas, 1994), business and finance (Calderon, 1993; Gerrard, Gibbons, \& Reis-Bergan, 1999; Kappes \& Sharot, 2015; Puri \& Robinson, 2007) and anti-social behaviour (e.g., reckless driving; DeJoy, 1999; White, Cunningham, \& Titchener, 2011). It is worth noting 
that Shah and colleagues concede that while they doubt the existence of a general optimism bias, some specific groups of people such as smokers and gamblers could have higher than average levels of optimism, suggesting that optimism is a trait rather than a pervasive cognitive bias. Nonetheless, given the widespread interest in unrealistic optimism and the recent alternative interpretation of the empirical findings, it is particularly important that tests are conducted to systematically investigate the robustness of the methods used to study optimism bias.

\subsection{Measuring Unrealistic Optimism}

1.2.1. The comparative method. Unrealistic optimism was traditionally measured by comparing participants' perceived chances of experiencing negative and positive life events with how likely they thought such events were to happen to people generally (Weinstein, 1980). Unrealistic optimism, or an optimism bias, is said to exist when people expect that their personal future outcomes are more favourable than would be predicted by a normative model of estimation (Harris \& Hahn, 2011; Mckay \& Dennett, 2009) and, indeed, results from studies using this 'comparative method' suggest that healthy people generally expect more positive and less negative things to happen to them than the average person (Shepperd et al., 2015).

The results of studies using this comparative method have come under attack though, in particular by Harris and Hahn (2011) who showed that unbiased simulated agents would produce seemingly optimistic answers when responding to questions used in the comparative method. Because the extant research showing optimism in humans was therefore suggested to be confounded, it was of great interest to academics on both side of this debate when a new methodology - one that did not rely on people's comparisons between themselves and the 
average person - not only provided additional evidence in support of a general optimism bias but also presented neuroscientific evidence to explain how this bias is maintained.

1.2.2. The belief update task. This relatively new research paradigm developed by Sharot, Korn and Dolan (2011; called the belief update task) has provided further evidence to support the existence of an optimism bias as well as a mechanistic account to explain how unrealistic optimism persists in the face of disconfirming evidence. Participants in this task are asked to estimate the likelihoods of certain adverse life events happening to them in the future and are subsequently presented with base rate statistics which display the probabilities of these events occurring to someone in the same sociocultural environment as them. In a second phase of the task, participants are asked to re-estimate their personal risk for each of the life events.

The amount that participants update their estimates in response to base rate feedback is used as a measure of how much they incorporate new information into their belief systems. Belief updates are compared across two trial types; trials in which participants receive desirable information and those in which they receive undesirable information. Trials are classified as 'desirable' or 'undesirable', depending on the valence of the event and whether the base rate is higher or lower than the participant's initial estimate (IE) of the likelihood of the event happening to them.

Several studies have shown that healthy participants generally update their beliefs more when base rates are desirable compared to undesirable (for a review, see Sharot and Garrett, 2016) and have concluded that this mechanism, whereby beliefs selectively update in response to desirable information, is responsible for pervasive unrealistic optimism. Using neuroimaging techniques, Sharot and colleagues have also shown dissociable patterns of neural activation in response to such desirable and undesirable information. For example, 
Sharot et al. (2011) found that desirable estimation errors were positively correlated with activation in the left inferior frontal gyrus (IFG), medial frontal cortex (MFC) and right cerebellum, whilst undesirable estimation errors were negatively correlated with activation in the right IFG. More crucially, activation in the right IFG differed as a function of individual differences in trait optimism. Individuals with high trait optimism scores exhibited reduced tracking of undesirable estimation errors in the right IFG relative to those with low scores. This latter finding suggests that trait optimism is linked to reduced neural coding of undesirable estimation errors. But despite the vast array of behavioural and neuroscientific evidence in support of the belief updating hypothesis (Garrett et al., 2014; Garrett \& Sharot, 2014; Kuzmanovic et al., 2015; Moutsiana et al., 2013, 2015; Sharot, Guitart-Massip et al., 2012; Sharot, Kanai et al., 2012, Sharot et al., 2011), there have also been some concerns expressed regarding the methodology that may invalidate these results (Harris et al., 2013; Shah et al., 2016).

1.2.2.1. Updating for positive life events. One critique made by Shah et al. (2016) is that the belief update task traditionally only asked participants to make estimates for negative life events. Sharot et al. (2011) asked participants to estimate the likelihood of events happening or not happening to them in an attempt to overcome this issue, but a Bayesian analysis conducted by Shah et al. (2016) showed that estimating the likelihood of a negative event not happening is not equivalent to estimating the likelihood of a positive event happening. They argued that if a biased task design, rather than biased participants, was causing seemingly optimistic updating, the reverse bias (i.e. a pessimism bias) should be observed in trials where positive events are used and the statistical design of the task is flipped. And, indeed, Shah et al. (2016) found a pessimistic pattern of updating when human participants were presented with positive events in this task, suggesting that optimistic updating is caused by a statistical artefact rather than a feature of human cognition. This 
finding is contentious, however, because a subsequent study did not find this reversal effect when using positive events and, in fact, showed optimistic updating for these stimuli too (Garrett \& Sharot, 2017).

It is also important to note that previous studies of comparative optimism have reported a 'valence effect'; participants were shown to exhibit more unrealistic optimism for negatively framed future events than positively framed events (Gold \& de Sousa, 2011). This finding suggests that people are more motivated to make biased judgements when thinking about negative compared to positive events - a conclusion that is consistent with loss aversion research which has shown that humans prefer to avoid losses rather than acquire gains and self-protect rather than self-enhance (Alicke \& Sedikides, 2009; Tversky \& Kahneman, 1985; Vohs \& Luce, 2010).

\subsubsection{Problems with randomly generated and skewed base rates. Garrett and} Sharot (2017) have suggested that caution should be taken when adapting the belief update task to study positive life events because there is a lack of accurate information regarding the likely frequency of such events. Shah et al. (2016) used bogus statistics in three of their five experiments, adding or subtracting a random percentage between $17 \%$ and $40 \%$ to or from participants' IEs in a uniform distribution.

Garrett and Sharot (2017) showed, using simulated data, that a skewed distribution of base rates will systematically and unequally affect belief updating, thus making it appear that people incorporate positive and negative information asymmetrically. Because people generally update more as estimation errors increase (Kuzmanovic et al., 2015; Sharot \& Garrett, 2016), any difference in the size of estimation errors across conditions in this task may lead to an artificial asymmetry in updating. As a result, it is vital either to make sure that 
base rates for positive and negative events are normally distributed around the same point or to appropriately control for estimation errors.

The formula used to generate base rate feedback in Shah et al.'s (2016) experiments was biased to produce larger estimation errors for more probable events because $17-40 \%$ of a large IE (e.g. $0.4 * 99=39.6)$ will necessarily produce a greater estimation error than $17-40 \%$ of a smaller IE (e.g. $0.4 * 1=0.4$ ). Therefore, if IEs were higher for desirable than undesirable trials we should expect to observe larger estimation errors for desirable trials and, thus, greater updating too. If the reverse were true and IEs were higher for undesirable information, estimation errors should be larger in undesirable than desirable trials, thus biasing participants to update more in response to undesirable than desirable information.

In Shah et al.'s (2016) experiments, participants tended to submit higher IEs for undesirable than desirable information when estimating the likelihood of positive events. The implications of this asymmetry are serious because an imbalance in estimation errors across conditions will likely exist if a biased base rate generator is used. And indeed, in all of Shah et al.'s (2016) experiments, estimation errors were greater in the positive event conditions when undesirable, as compared to desirable, information was presented. The average estimation error across their experiments was 10.33 (positive events $=10.47$; negative events $=10.19)$, meaning that after participants entered their IE they were shown a figure indicating the base rate was approximately 10 percentage points higher or lower than they first thought. However, there were marked differences depending on the desirability of the information. When positive events were presented, the average estimation error was 13.61 for undesirable information but only 7.33 for desirable information. The reverse pattern occurred for negative events, although to a lesser extent (desirable information $=12.07$; undesirable information $=$ 8.30). Without appropriately controlling for estimation errors, such differences will selectively influence update scores. 
It is important to note that in two of Shah et al.'s (2016) experiments the base rates were not experimentally manipulated. The base rates in these experiments were derived from externally sourced data (Harris, 2009), rather than the participant's IEs, and held constant across participants. The base rates in these two experiments were positively skewed however, due to the selection of events, to make the end result the same - estimation errors were much larger for undesirable than desirable information in positive event trials (and smaller in negative event trials). This outcome happens when base rates are positively skewed because estimation errors are small when the participants' IE is low and estimation errors are large when the participants' IE is high. Again, larger estimation errors for undesirable than for desirable information are produced for positive events, while the reverse is true for negative events (for more details, see Garrett \& Sharot, 2017).

\subsection{The Current Study}

Previous update task studies that have included positive events into the design have produced mixed findings. One of the aims of this study was to investigate whether a pessimistic pattern of belief updating would be observed for positive life events in this task, as found by Shah et al. (2016), or whether optimistic belief updating would be observed as was the case in Garrett and Sharot (2017).

These two previous studies included positive life events but differed in their design, which may explain the contrasting findings. Specifically, Garrett and Sharot (2017) conducted a pre-experimental survey to assess how often a selection of positive and negative events happened to people, on average, over the last four weeks and used the results of this survey to generate base rate feedback that was normally distributed around a mean of $50 \%$ in a belief update task undertaken by a separate group of participants. Their experiment was thus fundamentally different from the studies done by Shah et al. (2016) for three reasons: 
firstly, participants were asked to provide likelihood estimates for events that might occur in the next four weeks rather than over the course of a lifetime; secondly, the base rates were not randomly generated based on participants IEs but were instead based on the results of a preexperimental survey and held constant across participants; and thirdly, base rates were normally distributed around the 50\% midpoint of the scale for both negative and positive events.

The current study used the same experimental design as Shah et al. (2016), but half of the events (20 positive and 20 negative) used in their experiment were removed and, while the base rate statistics were still derived from participants' IEs, the probabilities presented to participants were calculated using absolute, rather than proportional, differences from the IEs to provide more balanced estimation errors.

In the current study, participants thought about and wrote down how or why the life event presented in each trial would happen to them or simply imagined and wrote down the event. After writing down each event, they completed a trial of the belief update task; they were asked to estimate how likely they thought the event was to happen to them, were subsequently presented with a (bogus) population base rate that indicated how prevalent the event was more generally, and were then immediately asked to revise their estimates (Kuzmanovic et al., 2015). The difference between people's IEs and their re-estimates acted as the dependent measure, and this update was compared across desirable and undesirable conditions for both positive and negative event trials.

We expected to observe the usual optimistically biased pattern of responding when participants thought about negative events and therefore hypothesised that belief updating would be greater for good news than for bad news in negative event trials. In the positive 
event trials, we hypothesised that there would be less optimistic belief updating than in the negative trials or possibly pessimistic belief updating as has been reported previously.

\section{METHOD}

\subsection{Participants}

Sixty participants were recruited through the UCL Psychology Subject Pool. Nine participants were excluded because they scored above the threshold for moderate depression (9) on the Patient Health Questionnaire-9 (PHQ-9). Thus, there were 51 participants (33 females and 18 males), aged between 18 and $58(M=24.90, S D=7.11)$. Participants were naïve to the specific purpose of the experiment and were simply told that the aim was to examine "people's judgments and perceptions of uncertain events." Participants all gave written informed consent and were compensated for their time. The study was approved by the UCL Department of Experimental Psychology Ethics Chair.

\subsection{Event Stimuli}

Short descriptions of 20 negative life events and 20 positive life events were used as event stimuli. The stimuli were a subset of those used by Shah et al. (2016) and relate to many different life domains (e.g. health, interpersonal relations, and random luck). Shah et al. used 80 rather than 40 stimuli, however pilot testing revealed that many of the events were perceived to be extremely likely to happen while others were not compatible with a priming manipulation that two-thirds of the participants were exposed to (the priming manipulation failed a manipulation check and the effects, although controlled for, are not reported in this paper). These events were therefore not used in this study. Average IEs showed that participants did not expect any of the events to occur with a likelihood lower than $10 \%$ or greater than $90 \%$ (see Appendix A).

\subsection{Design and Procedure}


This experiment used a 2 (event type: negative, positive) x 2 (information desirability: undesirable, desirable) within-subjects design. In sum, participants completed the belief update task, a funnelled debrief, stimulus evaluations and post-experimental measures in the UCL Psychology testing cubicles. Completion of the experiment took approximately $40 \mathrm{~min}$.

2.3.1. Belief update task. All 40 trials of the experimental task were completed in one block. Participants were presented with a written set of standardized instructions and a block of three practice trials (which consisted of events that were not used in the experimental phase). The belief update task was programmed and delivered using MATLAB Cogent 2000 toolbox (www.vislab.ucl.ac.uk/cogent.php).

Participants were presented with the series of life events one by one on a screen with a black background. Events were presented in white font and positioned in the centre of the screen. For each trial, they were asked to imagine the event happening to them in the future and instructed to write down on paper either the words on the screen (e.g. Miss a flight) or why or how life the event would happen to them. After this, the words, "Estimation of happening?" appeared on screen and participants were instructed to enter their IE - how likely they believed the event was to happen to them in the future - in the form of a two-digit numerical probability, using the keyboard. There was a time limit of $8 \mathrm{~s}$ for the initial estimation phase. Once the IE had been inputted (or the time limit was exceeded), a white fixation cross appeared on screen for $1 \mathrm{~s}$. Subsequently, the words "Average likelihood of happening" appeared on screen for $2 \mathrm{~s}$, accompanied by a base rate statistic which ostensibly showed the average likelihood among the general population. A fixation cross then reappeared for $1 \mathrm{~s}$ followed by the words "Re-estimation of happening?" and participants were given a further $8 \mathrm{~s}$ to input their second estimation. 
In contrast with Garrett and Sharot's (2017) procedure, in which true average base rates were presented to participants, base rates in this study were systematically manipulated. Specifically, statistics were derived from the participant's IEs; a random percentage between $3 \%$ and $25 \%$ (see, Kuzmanovic et al., 2015) was added to or subtracted from the participant's IEs. The base rate statistic provided participants with new, ostensibly objective, information that could help them to estimate how likely the event was to happen to them. The population base rate statistics were capped at $10 \%$ and $90 \%$ because very rare and very common events had purposefully been avoided.

2.3.2. Stimulus evaluation. A number of event characteristics, including familiarity, emotional arousal, valence, and base rate prevalence, have been found to modulate the optimism bias (e.g. Rose, Endo, Windschitl, \& Suls, 2008). Participants were therefore asked to provide ratings on 7-point scales for familiarity (from $1=$ completely unfamiliar to $7=$ completely familiar) and strength of valence (from $1=$ very negative to $7=$ very positive) for each event after the belief update task.

2.3.3. Post-experimental measures. Participants were asked to complete two postexperimental self-report measures: the Life Orientation Test Revised (LOT-R; Scheier, Carver, \& Bridges, 1994) and the Patient Health Questionnaire-9 (PHQ-9; Kroenke, Spitzer, \& Williams, 2001). The LOT-R measures trait optimism and consists of 10 items which are rated on a 5-point scale (e.g. "In uncertain times, I usually expect the best"; Cronbach's $\alpha=$ .79), with total scores ranging from 0 (pessimistic) to 24 (optimistic). The PHQ-9 is a measure of depression with total scores ranging from 0 (minimal depression) to 27 (severe depression). This scale was chosen for its brevity and because it is a well-validated measure that is widely used for measuring depression severity (Martin, Rief, Klaiberg \& Braehler, 2006). The PHQ-9 consists of 9 items that relate to depressive symptoms (e.g. Feeling down, depressed, or hopeless). Participants rate how often they have experienced these depressive 
symptoms over the past two weeks on a 4-point scale, from "0" (not at all) to "3" (nearly every day; Cronbach's $\alpha=.89$ ).

2.3.4. Debrief procedure. A funnelled debrief was used to give participants a chance to report any suppositions or doubts they had about the statistical information or the purpose of the study more generally. Answers to these questions showed that many of the participants deduced that belief updates were being measured, but none conjectured that belief updates might be dependent on the event type or desirability of the base rates, nor did they suspect that the base rates were false, although some claimed that they were "surprised" by certain statistics.

\subsection{Analysis}

Data were analysed using IBM SPSS Advanced Statistics 22.0 (International Business Machines Corporation, Armonk, NY). For each trial, estimation errors and belief update scores were calculated. Estimation errors were calculated as the difference between the participant's IE and the feedback given (by subtracting the IE from the base rate). As such, estimation errors were defined as positive when the base rate probability was greater than the IE and negative when the base rate probability was lower. Trials were classified as "desirable" in positive event trials when estimation errors were positive (i.e. something good is more likely than initially expected) and when estimation errors were negative in negative event trials (i.e. something bad is less likely than initially expected). The reverse was true for "undesirable" trials.

Belief updates were defined as the difference between the first and second probability estimates. These were calculated by subtracting the IE from the re-estimate (RE) when the estimation error was positive, and by subtracting the RE from the IE when the estimation error was negative. As a result, positive updates indicated a move towards the base rate 
probability regardless of event type or the desirability of the information, while negative scores indicated a move away from the base rate (see Appendix B).

In total, each of the participants $(N=51)$ gave two estimates for each of the 40 trials. Trials were excluded if the participant did not enter an estimate after the specified maximum time ( $8 \mathrm{~s}$ for each estimate). To reduce the impact of extreme updating and input mistakes, updates that exceeded a certain threshold $( \pm 3 \times$ the interquartile range) were classified as outliers and removed from the data (as in Shah et al., 2016).

Unlike any previous belief update task experiments (Garrett et al., 2014; Garrett \& Sharot, 2017; Kuzmanovic et al., 2015; Kuzmanovic, Rigoux, \& Vogeley, 2016; Moutsiana et al., 2013, 2015; Shah et al., 2016; Sharot, Guitart-Massip et al., 2012; Sharot, Kanai et al., 2012; Sharot et al., 2011; Sunstein, Bobadilla-Suarez, Lazzaro, \& Sharot, 2016), a linear mixed model (LMM) was used to analyse the data with update scores entered as the dependent variable. Information desirability (desirable/undesirable) and event type (positive event/negative event) were entered as fixed factors, subject as a random factor, and absolute estimation errors, strength of valence of events and familiarity of events entered as covariates. Two LMM procedures were carried out; the analysis was conducted using data from all 51 participants and was conducted again using only the control participants $(N=19)$ who were not exposed to the priming manipulation (described below). The results of a repeated-measures Analysis of Covariance (rmANCOVA; $N=51$ ) are also reported so that results can be compared with previous studies. In this latter analysis, a covariate controlling for the average difference in estimation errors across trial conditions (per participant) was included.

2.4.1. Controlling for a Priming Manipulation. A second aim of this study was to test whether optimistic belief updating is influenced by the level of construal at which 
information is processed. Two thirds of the participants in the current study wrote about either why or how life events would occur before being asked to provide probability estimates, as doing so was predicted to manipulate the level of construal, while the remaining third $(N=19)$ acted as a control group. Because a manipulation check showed that participants struggled to follow the instructions for the construal level manipulation, the data from all participants $(N=51)$ were collapsed and priming condition was accounted for in the first LMM and the rmANCOVA analyses. A second LMM analysis was also conducted, with participants from the priming conditions excluded $(N=19)$.

\section{RESULTS}

\subsection{Task Analysis}

3.1.1. Experimental Trials. Of the total 2,040 trials, 98 were excluded because participants either failed to input a response, entered an invalid response or entered extreme re-estimates. Following these exclusions, there was no significant difference in the number of trials between the positive $(n=972)$ and negative $(n=970)$ event conditions $(\chi 2(1,1941)=$ $.002, p=.96)$. There was, however, a significant difference between the number of trials in the desirable information $(n=873)$ and undesirable information $(n=1069)$ conditions $(\chi 2(1$, $1941)=19.78, p<.001)$. This difference also existed before any trials were excluded $(\chi 2(1$, $2019)=18.07, p<.001)$, suggesting that the base rate generator was 'forced' to produce more undesirable trials due to an imbalance in participants' IEs for positive and negative events.

3.1.2. Initial Estimates \& Estimation Errors. Participants' IEs were lower than the $50 \%$ midpoint for both negative events $(M=34.09, S D=24.65)$ and positive events $(M=$ $41.85, S D=29.96$ ). The IEs show that participants expected the positive events to occur more 
frequently than the negative events $(F(1,50)=21.82, \mathrm{p}<.001$; see Appendix $\mathrm{C}$ for more details).

Estimation errors were equal across the negative and positive event conditions $(F(1,50)=.47, \mathrm{p}=.50)$ and the desirable information and undesirable information conditions $(F(1,50)=3.63, \mathrm{p}=.062)$, but there was a significant interaction between these two conditions $(F(1,50)=22.76, \mathrm{p}<.001)$ showing that the prevalence of low IEs gave the base rate generator more 'room' for positive estimation errors than negative estimation errors (negative events, undesirable information: $M=14.39, S D=6.52$; negative events, desirable information: $M=12.44, S D=6.81$; positive events, undesirable information: $M=12.96, S D$ $=6.51$; positive events, desirable information: $M=14.30, S D=6.28$ ).

3.1.3. Base Rates. The base rate statistics were derived from participants' IEs, and, because average IEs were below 50\%, the distributions of base rates had a slight positive skew for both negative (skewness $=.69, \mathrm{SE}=.079 ;$ kurtosis $=-.50, \mathrm{SE}=.16$ ) and positive event conditions $($ skewness $=.33, \mathrm{SE}=.078$; kurtosis $=-.1 .24, \mathrm{SE}=.16$ ). The distribution of base rates was less skewed for positive events than for negative events, which is not surprising given that IEs were closer to $50 \%$ in the positive event condition, though the distribution for positive events had a heavier right tail as evidenced by the greater kurtosis.

\section{(Insert Figure 2)}

3.1.4. Event Evaluation. The positive events were rated as more familiar $(t(19)=$ $8.11, p<.001)$ and were considered to be weaker in their valence $(t(19)=7.06, p<.001)$ than the negative events. To account for this difference, strength of valence and familiarity of event were included as covariates in the LMM analysis.

\subsection{Linear Mixed Models}


3.2.1. Full data set. Asymmetric belief updating was observed: there was a main effect of information desirability, with participants updating their estimates more in response to desirable information $(M=6.37, S D=7.90)$ than undesirable information $(M=4.25, S D=$ 6.94; $F(1,1933)=40.58, p<.001)$. There was also a main effect of event valence, with participants updating their estimates more in response to negative events $(M=5.80, S D=$ 7.51) than positive events $(M=4.82, S D=7.36 ; F(1,1933)=6.52, p=.011)$, and a significant interaction effect between event type and desirability $(F(1,1933)=22.88, p<$ .001). A further analysis revealed that optimistic belief updating was exhibited for negative events (desirable information: $M=7.67, S D=8.38$; undesirable information: $M=3.92, S D=$ $6.83 ; F(1,1933)=59.50, p<.001)$ but not positive life events (desirable information: $M=$ 5.07, $S D=7.51$; undesirable information: $M=4.58, S D=7.10 ; F(1,1933)=1.10, p=.30)$. It is important to note, though, that when the covariates (EEs, familiarity of events and strength of valence of events) were not included in this model, or when IEs were controlled for instead of EEs (as recommended by Shah et al., 2016), optimistic updating was observed for positive events, as well as for negative events.

Control condition. When the participants in the priming conditions were excluded $(N$ =19), a similar pattern was observed: there was a significant main effect of information desirability $(F(1,717)=29.22, p<.001)$ and a significant interaction between event type and information desirability $(F(1,717)=10.10, p=.002)$, with participants updating more in response to desirable information $(M=7.68, S D=8.64)$ than undesirable information $(M=$ 3.01, $S D=5.46)$ for negative life events $(F(1,717)=34.80, p<.001)$ but not for positive life events (desirable information: $M=5.41, S D=7.61$; undesirable information: $M=4.22, S D=$ $5.46 ; F(1,717)=2.49, p=.12)$. There was, however, no main effect of event type $(F(1,717)=$ $.747, p=.39)$. Again, when the covariates were not included in this model, or when IEs were controlled for instead of EEs, optimistic updating was observed for positive events too. 


\subsection{Repeated-Measures ANCOVA}

Comparing event type and information desirability at the condition level rather than by trial, as has been done in the past, also produced similar results. With all participants $(N=$ 51) included, there was a significant main effect of information desirability (desirable information: $M=6.61, S D=4.09$; undesirable information: $M=4.32, S D=2.93 ; F(1,47)=$ $12.35, p=.001$ ), a main effect of event type (positive events: $M=4.92, S D=3.02$; negative events: $M=6.01, S D=3.44 ; F(1,47)=5.77, p=.02)$ but no interaction between event type and information desirability $(F(1,47)=1.26, p=.27)$.

\section{(Insert Figure 3)}

\section{DISCUSSION}

Shah et al. (2016) have claimed that including positive life events should reveal a fundamental flaw in the belief update task. In the current study, however, this was not the case. The results presented here suggest that people are optimistically biased when updating beliefs for negative but not positive events; the flip in asymmetric updating across negative and positive events observed by Shah et al. was not replicated in the current study. Consistent with previous theories of unrealistic optimism (Garrett et al., 2014; Kuzmanovic et al., 2015; Sharot et al., 2011; Shepperd et al., 2015; Weinstein, 1980), the evidence suggests that people selectively incorporate desirable information about their future into their beliefs while, by comparison, largely disregarding undesirable information. Overall, the results presented here are therefore more similar to those of Garrett and Sharot (2017) than of Shah et al. (2016), despite using the same methodology as Shah et al. (2016) with only small adjustments to the set of events included and the formula used to generate base rate feedback.

Shah et al. (2016) claim that if biased updating is in fact caused by a statistical artefact, then including positive events into this task should result in unbiased updating 
overall as the effects on updating across the two event type conditions should cancel each other out. Essentially, if the task is biased to produce greater updating for negative than positive estimation errors, we should expect to see optimistic updating for negative events and pessimistic updating for positive events. The fact that this does not happen suggests that optimistic updating for negative events is not due to a flawed methodology but is the result of a cognitive bias. We did not, however, observe optimistic updating for positive events, as has been reported elsewhere (Garrett \& Sharot, 2017), suggesting that more research needs to be conducted to understand when and why people exhibit biased probability updating when given new information about the likelihood of future positive life events.

\subsection{Initial Estimates, Base Rates and Estimation Errors}

The population base rates that were presented to participants in this study were derived from their IEs, as in other recent belief update task experiments (Kuzmanovic et al., 2015; Shah et al., 2016), due to the lack of accurate information regarding the likely frequency of positive events. This contrasts with the traditional approach used by Sharot et al. (2011) of providing genuine population statistics (about negative events) sourced from online resources (e.g. the Office for National Statistics). Care must be taken when deciding how to select and control for the base rate statistics because differences across conditions can affect the magnitude of belief updating at the group level. In the current experiment, even though the base rates were false, the formula used to generate these statistics was designed to reduce such differences.

In this experiment, base rates and IEs were both positively skewed while estimation errors, though generally equivalent across the event type and information desirability conditions, were greater when base rates were higher than IEs (i.e., positive estimation errors). The base rate generator was unable to produce large negative estimation errors for 
low IEs (e.g., 20\%) because base rates were capped at 10\%. Therefore, for IEs of $20 \%$, the base rate generator would either produce a small negative estimation error (between 3-10\%) or would produce a positive estimation error of any size (between 3-25\%). Note, though, that the differences between conditions were relatively small because the base rate statistics were calculated using absolute, rather than proportional, differences from participants' IEs and differences in estimation errors were properly controlled for, by trial rather than condition, in the LMM analyses.

The main difference between our paradigm and that of Shah et al (2016) is that Shah et al. generated base rates by adding or subtracting a random percentage between $17 \%$ and $40 \%$ of the IE to or from participants' IEs instead of adding and subtracting absolute percentages. Doing so will necessarily lead to unequal estimation errors when IEs are larger in one condition than another, with larger estimation errors for IEs that are at the higher end of the scale (i.e., very commonly occurring events). In our study, absolute percentage increases and decreases of 3\%-25\% were used to generate the base rate statistics in order to avoid this pitfall. Given the imbalance of estimation errors in Shah et al.'s experiments and the confounding effects that these have on belief updates, it seems that the adaptations to the base rate generator and the event stimuli (which affect participants' IEs and thus the base rates generated) in the current experiment can explain the difference in results.

\subsection{Statistical Analysis Procedure}

The LMM approach was taken, rather than the more commonly used rmANCOVA, because it is more appropriate to analyse updates by event rather than condition and control for estimation errors by trial rather than controlling for average differences in estimation errors across conditions. This was the first belief update task experiment to use the LMM procedure, although two recent studies have used a trial-by-trial analysis (Garrett \& Sharot, 
2017; Kuzmanovic et al., 2016). Note however that although it is possible to measure updates using two general linear models, one for negative life events and one for positive life events, this method requires an additional test, the analysis will be subject to multiple comparisons, and higher significance thresholds will need to be applied. Furthermore, the general linear models analysis does not produce results for the interaction effect between event type and information desirability. The LMM procedure therefore offers a cleaner and more precise approach than the other two analyses that have been used previously.

Of note, in the current study the interaction effect between event type and information desirability reported for the LMM analyses did not hold when using the rmANCOVA. As stated previously, the LMM procedure allows the researcher to analyse data by trial rather than across conditions. This enhanced control should therefore provide a more fine-grained analysis and detect effects that the rmANCOVA does not. The fact that this interaction was not significant in the rmANCOVA analysis, even though unbiased updating was observed for positive events, provides strong evidence that more precise findings can be obtained when using the LMM procedure for this task.

\subsection{Theoretical Contributions and Future Research}

Future studies should seek to disentangle if and when people exhibit biased belief updating in response to new information about the likelihood of future positive events. In contrast to Garrett and Sharot (2017), the results presented here suggest that positive and negative events have different effects on motivated reasoning and biased learning. This finding is consistent with much of the extant literature: Gold and de Sousa (2011) have previously argued that predictions about positive events are less biased than negative events, while previous well-documented psychological theories have suggested that humans have a stronger desire to avoid economic losses than acquire gains and, with regard to self-identity, 
self-protect rather than self-enhance (Alicke \& Sedikides, 2009; Tversky \& Kahneman, 1985; Vohs \& Luce, 2010). It seems that the same principle may apply when humans think about personal future outcomes.

Given the previous theoretical contributions, we would speculate that a difference in motivated reasoning and biased information integration for positive and negative events may be due to the asymmetric consequences that positive and negative events are likely to have had on human life over the course of evolution. Indeed, a lack of pleasurable events in one's future may be disappointing but is unlikely to be fatal. However, experiencing a truly negative event, for most of human history, would have likely resulted in an increased risk of death. Appropriately or overly focusing on new information about the increased likelihood of such tragedies can trigger feelings of hopelessness, depression and behavioural inhibition, and thus hinder productive daily functioning (Garrett et al., 2014; Sharot, 2011; Varki, 2009). Thus, humans may have developed a selective bias to prevent such a decrease in motivation and productivity.

It is unclear why these findings differ from those reported by Garrett and Sharot (2017), but, as mentioned earlier, their experiment was fundamentally different from the one presented here for three reasons. Garrett and Sharot asked participants to estimate the probability of events occurring in the next four weeks rather than over the course of a lifetime, provided them with genuine base rate information rather than bogus statistics generated based on the participants' IE, and used base rates that were normally distributed around the $50 \%$ midpoint of the scale. It is therefore possible that humans update beliefs in an optimistic manner when given new information about concrete, temporally proximal future positive events but do not do so for positive events that may occur sometime in the distant future. It is also possible that participants were unbiased for positive events in our study because they were more suspicious of the base rates presented in these trials than they were 
for negative event trials. Even though participants did not report any explicit suspicions about the truth of the base rates, if participants believed that the base rate feedback presented for positive events was not credible they may have given less weight to these statistics and thus updated less in these trials.

Finally, there are still other questions remaining about the methodology used in the belief update task that need to be resolved. Shah et al. (2016) point out that the desirability of base rate information cannot be assessed without knowledge of the participant's previous estimate of the base rate because there are in fact two ways that a person can receive new information about their personal risks: learning new information about population base rates or learning new diagnostic information that will affect their personal vulnerability. When estimating personal risk, both types of information should be considered. Consequently, participants in the update task may have additional diagnostic information regarding their own personal risk which is not presented to the researchers but does affect their estimations. While other studies have shown that accounting for the difference between participants' initial estimates of the average base rates and the base rates provided by the experimenter does not alter the observed pattern of optimistic updating (Garrett \& Sharot, 2014;

Kuzmanovic et al., 2015), more work is needed to better understand how much the credibility of the base rates and the diagnosticity of evidence of personal vulnerability affects belief updating.

In conclusion, this study has provided evidence to support the existence of a general and pervasive optimism bias, which adds to the current debate around the robustness of the methodologies used to measure unrealistic optimism. In particular, the statistical artefact account of the belief update task's findings was challenged. The current study showed that changing the way that base rate information (about the occurrences of life events) is generated in order to minimize any imbalances in estimation errors across conditions results 
in an optimistic pattern of belief updating for negative but not positive events. The results may, therefore, signal the presence of a valence-dependent evolutionary cognitive mechanism. 


\section{Figure Legends}

Figure 1: The Belief Update Task. The time-course of a single experimental trial in the belief update task. Each trial began with the presentation of one of the 40 life events and participants were asked to write down the words displayed. Participants were given unlimited time to write the event. Following this, they were requested to estimate the likelihood of the event occurring at some point in their lives (duration $8 \mathrm{~s}$ ). Following an inter-stimulus interval of $1 \mathrm{~s}$, participants were presented with a population base rate figure $(2 \mathrm{~s})$. An inter-stimulus interval of $1 \mathrm{~s}$ intervened, then participants were asked to provide a re-estimate $(8 \mathrm{~s})$. In this example, a negative event is shown, the base rate is lower than initial estimate and the information presented is classified as desirable information. The update in this example is eight percentage points. Maximum durations, in seconds, are provided below the screen displays.

Figure 2: Distribution of the base rate statistics presented to participants. The base rates were positively skewed for both negative and positive events.

Figure 3: Bar graph showing mean update scores as a function of life event (positive or negative). Plots show desirable (red bars) and undesirable (blue bars) information. (A) The full data set was analysed using the Linear Mixed Model procedure. (B) The control condition was analysed using the Linear Mixed Model procedure. (C) The full data set was analysed using the repeated measures Analysis of Covariance procedure. Error bars use the standard error of the mean. 


\section{Acknowledgements}

We would like to thank Neil Garrett and Tali Sharot for providing a previous version of the MATLAB code for the Belief Update Task and providing comments on the manuscript. We would also like to thank Adam Harris and Punit Shah for providing a list of event stimuli and Toby Pilditch for helping to develop the base rate generator. The project was conceived by both authors. JM designed, conducted and analysed the experiment and drafted the manuscript. SB commented on the manuscript.

\section{Funding}

This research did not receive any specific grant from funding agencies in the public, commercial, or not-for-profit sectors. 


\section{References}

Alicke, M. D., \& Sedikides, C. (2009). Self-enhancement and self-protection: What they are and what they do. European Review of Social Psychology, 20, 1-48. http://dx.doi.org/10.1080/10463280802613866

Calderon, T. G. (1993). Predictive properties of analysis' forecasts of corporate earnings. The Mid-Atlantic Journal of Business, 29, 41-59.

DeJoy, D. M. (1989). The optimism bias and traffic accident risk perception. Accident Analysis \& Prevention, 21, 333-340. http://dx.doi.org/10.1016/0001-4575(89)90024-9

Dillard, A. J., McCaul, K. D., \& Klein, W. M. (2006). Unrealistic optimism in smokers: Implications for smoking myth endorsement and self-protective motivation. Journal of Health Communication, 11, 93-102.

Garrett, N., \& Sharot, T. (2014). How robust is the optimistic update bias for estimating selfrisk and population base rates? PloS One, 9, e98848.

http://doi.org/10.1371/journal.pone.0098848

Garrett, N. \& Sharot, T. (2017). Optimistic update bias holds firm: Three tests of robustness following Shah et al. Consciousness and Cognition, 50, 12-22. doi: 10.1016/j.concog.2016.10.013

Garrett, N., Sharot, T., Faulkner, P., Korn, C. W., Roiser, J. P., \& Dolan, R. J. (2014). Losing the rose tinted glasses: neural substrates of unbiased belief updating in depression. Frontiers in Human Neuroscience, 8, 1-9. doi: 10.3389/fnhum.2014.00639

Gerrard, M., Gibbons, F. X., \& Reis-Bergan, M. (1998). The effect of risk communication on risk perceptions: the significance of individual differences. Journal of the National Cancer Institute Monographs, 25, 94-100. http://jncimono.oxfordjournals.org/ 
Gold, R. S., \& de Sousa, P. N. (2011). When does event valence affect unrealistic optimism? Psychology, Health \& Medicine, 17, 105-115. doi: $10.1080 / 13548506.2011 .582503$

Harris, A. J. L., \& Hahn, U. (2011). Unrealistic optimism about future life events: A cautionary note. Psychological Review, 118, 135-154. doi: 10.1037/a0020997

Harris, A. J. L., Shah, P., Catmur, C., Bird, G., \& Hahn, U. (2013). Autism, optimism and positive events: Evidence against a general optimistic bias. In M. Knauff, M. Pauen, N. Sebanz, \& I. Wachsmuth (Eds.), Proceedings of the $35^{\text {th }}$ Annual Conference of the Cognitive Science Society. Austin, TX: Cognitive Science Society.

Jansen, L. A., Appelbaum, P. S., Klein, W. M., Weinstein, N. D., Cook, W., Fogel, J. S., \& Sulmasy, D. P. (2011). Unrealistic optimism in early-phase oncology trials, Irb, 33, 18.

Kappes, A., \& Sharot, T. (2015). Optimism and entrepreneurship: A double-edged sword. Nesta report, February. https://www.nesta.org.uk/sites/default/files/optimism_and_entrepreneurship_a_double-edged_sword.pdf

Korn, C. W., Sharot, T., Walter, H., Heekeren, H. R., \& Dolan, R. J. (2014). Depression is related to an absence of optimistically biased belief updating about future life events. Psychological Medicine, 44, 579-592.

Krieger, J. L., Murray, F., Mountain, J., Moreno, T., Carere, A. D., Roberts, S., \& Green, R. (2014). Risk perception and medical utilization: Evidence from the PGen study. Presented at the American College of Medical Genetics and Genomics (ACMG), American College of Medical Genetics and Genomics (ACMG). 
Kroenke, K., Spitzer, R. L., \& Williams, J. B. W. (2001). The PHQ-9. Journal of General Internal Medicine, 16, 606-613. doi: 10.1046/j.1525-1497.2001.016009606.x

Kuzmanovic, B., Jefferson, A., \& Vogeley, K. (2015). Self-specific Optimism Bias in Belief Updating Is Associated with High Trait Optimism. Journal of Behavioral Decision Making, 28, 281-293. doi: 10.1002/bdm.1849

Kuzmanovic, B., Rigoux, L., \& Vogeley, K. (2016). Brief report: Reduced optimism bias in self-referential belief updating in high-functioning autism. Journal of Autism and Developmental Disorders, 46, 1-9. doi: 10.1007/s10803-016-2940-0

Martin, A., Rief, W., Klaiberg, A., \& Braehler, E. (2006). Validity of the brief patient health questionnaire mood scale (PHQ-9) in the general population. General Hospital Psychiatry, 28, 71-77. doi: 10.1016/j.genhosppsych.2005.07.003

McKay, R. T., \& Dennett, D. C. (2009). The evolution of misbelief. Behavioral and Brain Sciences, 32, 493-510. doi: 10.1017/S0140525X09990975

Moutsiana, C., Garrett, N., Clarke, R. C., Lotto, R. B., Blakemore, S. J., \& Sharot, T. (2013). Human development of the ability to learn from bad news. Proceedings of the National Academy of Sciences, 110, 16396-16401. doi: 10.1073/pnas.1305631110

Moutsiana, C., Charpentier, C. J., Garrett, N., Cohen, M. X., \& Sharot, T. (2015). Human frontal-subcortical circuit and asymmetric belief updating. The Journal of Neuroscience, 35, 14077-14085. doi: 10.1523/JNEUROSCI.1120-15.2015

Puri, M., \& Robinson, D. T. (2007). Optimism and economic choice. Journal of Financial Economics, 86(1), 71-99. http://dx.doi.org/10.1016/j.jfineco.2006.09.003

Rose, J. P., Endo, Y., Windschitl, P. D., \& Suls, J. (2008). Cultural differences in unrealistic optimism and pessimism: The role of egocentrism and direct versus indirect 
comparison measures. Personality and Social Psychology Bulletin, 34, 1236-1248. doi: $10.1177 / 0146167208319764$

Shah, P., Harris, A. J. L., Hahn, U., Catmur, C. \& Bird, G. A pessimistic view of optimistic belief updating. Cognitive Psychology, 90, 71-127. doi:

10.1016/j.cogpsych.2016.05.004

Sharot, T. (2011). The optimism bias. Current Biology, 21, 941-945. http://dx.doi.org/10.1016/j.cub.2011.10.030

Sharot, T. (2012). The optimism bias: Why we're wired to look on the bright side. London, UK: Constable \& Robinson Limited.

Sharot, T., \& Garrett, N. (2016). Forming beliefs: Why valence matters. Trends in Cognitive Sciences, 20, 25-33. http://doi.org/10.1016/j.tics.2015.11.002

Sharot, T., Guitart-Masip, M., Korn, C. W., Chowdhury, R., \& Dolan, R. J. (2012). How dopamine enhances an optimism bias in humans. Current Biology, 22, 1477-1481. http://dx.doi.org/10.1016/j.cub.2012.05.053

Sharot, T., Kanai, R., Marston, D., Korn, C. W., Rees, G., \& Dolan, R. J. (2012). Selectively altering belief formation in the human brain. Proceedings of the National Academy of Sciences of the United States of America, 109, 17058-17062.

Sharot, T., Korn, C. W., \& Dolan, R. J. (2011). How unrealistic optimism is maintained in the face of reality. Nature Neuroscience, 14, 1475-1479. doi:10.1038/nn.2949

Scheier, M. F., Carver, C. S., \& Bridges, M. W. (1994). Distinguishing optimism from neuroticism (and trait anxiety, self-mastery, and self-esteem): A reevaluation of the Life Orientation Test. Journal of Personality and Social Psychology, 67, 1063-1078. http://dx.doi.org/10.1037/0022-3514.67.6.1063 
Shepherd, R. (2002). Resistance to changes in diet. Proceedings of the Nutrition Society, 61, 267-272. doi: 10.1079/PNS2002147

Shepperd, J. A., Waters, E. A., Weinstein, N. D., \& Klein, W. M. (2015). A primer on unrealistic optimism. Current Directions in Psychological Science, 24, 232-237. doi: $10.1177 / 0963721414568341$

Strunk, D. R., Lopez, H., \& DeRubeis, R. J. (2006). Depressive symptoms are associated with unrealistic negative predictions of future life events. Behaviour Research and Therapy, 44, 861-882.

Sunstein, C. R., Bobadilla-Suarez, S., Lazzaro, S. C., \& Sharot, T. (2016). How people update beliefs about climate change: Good news and bad news. Available at SSRN: 2821919.

Taylor, S. E., \& Brown, J. D. (1988). Illusion and well-being: A social psychological perspective on mental health. Psychological Bulletin, 103, 193-210. doi: 10.1037/0033-2909.103.2.193

Trope, Y. (1980). Self-assessment, self-enhancement, and task preference. Journal of Experimental Social Psychology, 16, 116-129.

Tversky, A., \& Kahneman, D. (1974). Judgment under uncertainty: Heuristics and biases. Science, 185, 1124-1131. doi: 10.1007/978-94-010-1834-0_8

Tversky, A., \& Kahneman, D. (1985). The framing of decisions and the psychology of choice. Science, 211, 453-458. 10.1007/978-3-642-70634-9_6

Tyler, J. M., \& Rosier, J. G. (2009). Examining self-presentation as a motivational explanation for comparative optimism. Journal of Personality and Social Psychology, 97, 716-727. http://dx.doi.org/10.1037/a0016231 
van der Velde, F. W., Hooykaas, C., \& van der Joop, P. (1992). Risk perception and behavior: Pessimism, realism, and optimism about aids-related health behavior. Psychology \& Health, 6, 23-38. doi: 10.1080/08870449208402018

van der Velde, F. W., van der Joop, P., \& Hooykaas, C. (1994). Perceiving AIDS related risk: Accuracy as a function of differences in actual risk. Health Psychology, 13, 25-33. doi: $0.1037 / 0278-6133.13 .1 .25$

Varki, A. (2009). Human uniqueness and the denial of death. Nature, 460, 684-684. doi: $10.1038 / 460684 c$

Vohs, K. D., \& Luce, M. F. (2010). Judgment and decision making. In R. F. Baumeister \& E. J. Finkel (Eds.), Advanced social psychology: The state of the science (pp. 733-756). Oxford, England: Oxford University Press.

Weinstein, N. D. (1980). Unrealistic optimism about future life events. Journal of Personality and Social Psychology, 39, 806.

Weinstein, N.D. \& Klein, W.M. (1995). Resistance of personal risk perceptions to debiasing interventions. Health Psychology, 14, 132-140.

White, M. J., Cunningham, L. C., \& Titchener, K. (2011). Young drivers' optimism bias for accident risk and driving skill: Accountability and insight experience manipulations. Accident Analysis \& Prevention, 43, 1309-1315.

http://dx.doi.org/10.1016/j.aap.2011.01.013 



\section{Appendix A}

Table 1: Full list of the 40 event stimuli. Participants mean (M) initial estimates for each event in the belief update task (standard deviations (SD) are provided).

\begin{tabular}{|c|c|c|}
\hline \multirow[b]{2}{*}{ Event Stimuli } & \multicolumn{2}{|c|}{$\underline{\text { Initial Estimates }}$} \\
\hline & $M$ & $S D$ \\
\hline Accidentally putting an electronic device in the washing machine & 26.15 & 23.90 \\
\hline Being arrested & 15.10 & 19.06 \\
\hline Finding your partner cheating on you & 37.40 & 22.42 \\
\hline Finding yourself in unmanageable debt & 20.98 & 15.44 \\
\hline Being sworn at in public & 52.88 & 30.04 \\
\hline Bone fracture & 35.91 & 25.65 \\
\hline Domestic burglary & 32.96 & 19.99 \\
\hline Falling down stairs & 43.62 & 25.65 \\
\hline Food poisoning with need to visit a doctor & 43.30 & 27.06 \\
\hline Fraud when buying something on the internet & 38.46 & 22.37 \\
\hline Get trapped in a lift & 26.57 & 20.81 \\
\hline Having a stroke & 28.90 & 18.26 \\
\hline House vandalised & 25.24 & 20.18 \\
\hline Losing mobile phone & 45.73 & 27.86 \\
\hline Losing wallet & 40.00 & 26.44 \\
\hline Miss a flight & 34.41 & 26.71 \\
\hline Theft from your vehicle & 28.82 & 18.35 \\
\hline Vehicle (car/ bike) stolen & 36.10 & 27.26 \\
\hline Victim of bullying at work (nonphysical) & 35.13 & 22.13 \\
\hline Victim of mugging & 32.19 & 18.78 \\
\hline Attending a family member's wedding & 74.48 & 25.78 \\
\hline Receiving a standing ovation from a large audience & 34.42 & 25.93 \\
\hline Buying a new house & 57.84 & 31.33 \\
\hline Maintaining a constant healthy weight for 10 years & 57.91 & 30.74 \\
\hline Finding $£ 10$ or more on the street & 39.00 & 29.21 \\
\hline
\end{tabular}


Getting a large bonus payment at work

Getting a new pet animal

Going to a movie premiere

Having a book/article published

Having a healthy child

Making a profit when selling a valuable item

Meeting a member of the royal family

Raise over $£ 1000$ for charity

Seeing someone famous while on public transport

Upgraded to first class on a flight

Winning a prize in a media (TV/radio/newspaper) competition

Winning a race

Winning a raffle at a fairground

Winning an award in recognition of your work

Your achievements in newspaper

Events used during the training session

Dying before 90

$\mathrm{n} / \mathrm{a}$

$\mathrm{n} / \mathrm{a}$

Glaucoma

$\mathrm{n} / \mathrm{a}$

$\mathrm{n} / \mathrm{a}$

Post-traumatic stress disorder

$\mathrm{n} / \mathrm{a}$ $\mathrm{n} / \mathrm{a}$ 


\section{Appendix B}

The formula used to calculate estimation errors was as follows. IE is participants' initial probability estimate. BR is the average base rate statistic that was presented after participants made their first estimate. EE is the estimation error.

$$
\mathrm{BR}-\mathrm{IE}=\mathrm{EE}
$$

The formula used to calculate belief updates was as follows (RE is participants' updated reestimate):

For Positive EEs: RE - IE = Update

For Negative EEs: IE $-\mathrm{RE}=$ Update

Table 1: Categorisation of events.

\begin{tabular}{lccc}
\hline & $\begin{array}{c}\text { Positive EE } \\
(\mathrm{BR}>\mathrm{IE})\end{array}$ & & $\begin{array}{c}\text { Negative EE } \\
(\mathrm{BR}<\mathrm{IE})\end{array}$ \\
\cline { 2 - 3 } Positive Event & Desirable Information & & Undesirable Information \\
Negative Event & Undesirable Information & & Desirable Information \\
\hline
\end{tabular}




\section{Appendix C}

Table 2: Descriptive statistics for participants' probability estimates and the base rates presented by event type and information desirability. IE is the average initial estimate of how likely events are to occur to them. BR is the average base rate for events happening, as calculated by the base rate generator. RE is the updated re-estimate provided after participants have seen the base rate statistic. Figures show mean averages (standard deviations).

\begin{tabular}{|c|c|c|c|c|c|c|}
\hline & \multicolumn{3}{|c|}{ Positive Events } & \multicolumn{3}{|c|}{ Negative Events } \\
\hline & $\begin{array}{c}\text { Undesirable } \\
\text { Info }\end{array}$ & $\begin{array}{l}\text { Desirable } \\
\text { Info }\end{array}$ & Overall & $\begin{array}{l}\text { Undesirable } \\
\text { Info }\end{array}$ & $\begin{array}{c}\text { Desirable } \\
\text { Info }\end{array}$ & Overall \\
\hline IE & $\begin{array}{c}62.78 \\
(25.85)\end{array}$ & $\begin{array}{c}26.19 \\
(22.30)\end{array}$ & $\begin{array}{c}41.85 \\
(29.96)\end{array}$ & $\begin{array}{c}25.32 \\
(19.28)\end{array}$ & $\begin{array}{l}52.15 \\
(24.70)\end{array}$ & $\begin{array}{c}34.09 \\
(24.65)\end{array}$ \\
\hline BR & $\begin{array}{c}49.82 \\
(24.64)\end{array}$ & $\begin{array}{c}40.49 \\
(21.86)\end{array}$ & $\begin{array}{c}44.48 \\
(23.54)\end{array}$ & $\begin{array}{c}39.71 \\
(19.59)\end{array}$ & $\begin{array}{c}39.70 \\
(22.60)\end{array}$ & $\begin{array}{c}39.71 \\
(20.61)\end{array}$ \\
\hline $\mathrm{RE}$ & $\begin{array}{c}58.68 \\
(28.43)\end{array}$ & $\begin{array}{c}31.55 \\
(25.29)\end{array}$ & $\begin{array}{c}43.16 \\
(29.86)\end{array}$ & $\begin{array}{c}29.64 \\
(21.48)\end{array}$ & $\begin{array}{c}44.80 \\
(27.15)\end{array}$ & $\begin{array}{c}34.59 \\
(24.53)\end{array}$ \\
\hline
\end{tabular}




\section{Appendix D}

Table 3: Stimulus evaluations provided by participants after the belief update task. Familiarity $(1=$ low to $7=$ high $)$ and strength of valence ratings $(1=$ very negative to $7=$ very positive $)$ were assessed using a subjective, post-task questionnaire.

\begin{tabular}{|c|c|c|c|c|}
\hline \multirow[b]{2}{*}{ Type of Information } & \multicolumn{2}{|c|}{$\begin{array}{l}\text { Positive Events } \\
\text { Mean (SD) }\end{array}$} & \multicolumn{2}{|c|}{$\begin{array}{l}\text { Negative Events } \\
\text { Mean (SD) }\end{array}$} \\
\hline & Undesirable & Desirable & Undesirable & Desirable \\
\hline Familiarity & $\begin{array}{l}4.11 \\
(0.76)\end{array}$ & $\begin{array}{l}3.77 \\
(0.64)\end{array}$ & $\begin{array}{l}3.13 \\
(0.64)\end{array}$ & $\begin{array}{c}3.40 \\
(0.61)\end{array}$ \\
\hline Strength of Valence & $\begin{array}{c}5.97 \\
(0.45)\end{array}$ & $\begin{array}{c}5.80 \\
(0.49)\end{array}$ & $\begin{array}{c}1.77 \\
(0.33)\end{array}$ & $\begin{array}{c}1.89 \\
(0.32)\end{array}$ \\
\hline
\end{tabular}

*Note, all positive and negative events were perceived as positive and negative, respectively. 
Table 4: The Patient Health Questionnaire-9 (PHQ-9).

Participants were asked "Over the last 2 weeks, how often have you been bothered by any of the following problems?

Little interest or pleasure in doing things

Feeling down, depressed, or hopeless

Trouble falling or staying asleep, or sleeping too much

Feeling tired or having little energy

Poor appetite or overeating

Feeling bad about yourself — or that you are a failure or have let yourself or your family down

Trouble concentrating on things, such as reading the newspaper or watching television Moving or speaking so slowly that other people could have noticed? Or the opposite being so fidgety or restless that you have been moving around a lot more than usual Thoughts that you would be better off dead or of hurting yourself in some way

Note: The PHQ-9 was originally developed by Kroenke, Spitzer and Williams (2001) and is commonly used in clinical settings to measure depression severity. 


\section{Appendix F}

Table 5: The Life Orientation Test-Revised (LOT-R) scale.

Participants were asked to describe how much they agreed with the following statements

In uncertain times, I usually expect the best.

It's easy for me to relax

If something can go wrong for me, it will.

I'm always optimistic about my future.

I enjoy my friends a lot

It's important for me to keep busy

I hardly ever expect things to go my way

I don't get upset too easily

I rarely count on good things happening to me. yourself in some way

Overall, I expect more good things to happen to me than bad.

Note: The LOT-R was developed by Scheier, Carver and Bridges (1994). Items 2, 5, 6, and 8 are fillers. Items 3, 7 and 9 are reverse coded. 